\title{
Embriología y «ciencia oficial»: la aportación de la escuela anatómica de José Escolar a la embriología durante el primer Franquismo (1939-1959)
}

\author{
Raúl Velasco Morgado (*) \\ (*) orcid.org/0000-0002-6958-9090. Área de Historia de la Ciencia. Universidad de Salamanca. \\ raulvmorgado@hotmail.com
}

Dynamis

[0211-9536] 2015; 35 (1): 153-175

http://dx.doi.org/10.4321/S0211-95362015000100007
Fecha de recepción: 2 de agosto de 2013

Fecha de aceptación: 10 de abril de 2014

SUMARIO: 1.-Introducción. 2.-José Escolar, creador y organizador de una escuela anatómica. 3.-Los comienzos. 4.-La «Sección de anatomía» del Instituto Cajal. 5.-La embriología y la visión alemana de la neuroanatomía. 6.-Conclusiones.

RESUMEN: En el presente trabajo se hace un análisis de la aportación de la escuela anatómica de José Escolar (1913-1998) a la embriología durante las dos primeras décadas de la dictadura franquista. Se hace un especial énfasis en el proceso por el que, gracias al apoyo del naciente CSIC, el grupo español contactó con la morfología alemana que estaba desarrollando Hugo Spatz (1888-1979) en el Max Planck-Institut für Hirnforschung. A través de nuestro estudio se constatan las diversas influencias que terminaron por dibujar la anatomía y embriología de Escolar. En España, detectamos una influencia directa de la morfología gegenbauriana de Gumersindo Sánchez-Guisande (1894-1976) así como de la neuroanatomía de Juan José Barcia Goyanes (1901-2003), llena de referencias a la obra de Braus. Los contactos internacionales de los «escolarianos» — con la ciencia estadounidense primero y posteriormente con la alemana- constituyeron un grupo de trabajo homogéneo con una anatomía única (funcional y ontofilogénetica) pero con unos intereses investigadores tan amplios que obligaron a subespecializarse al equipo. Fruto de una intensa relación con la comunidad anatómica alemana, durante los años 50 fueron apareciendo una serie de importantes trabajos embriológicos entre los que destacan los que realizó Escolar sobre el desarrollo de la amígdala y el allocórtex, los de Fernando Reinoso sobre la embriología del diencéfalo y los descubrimientos de Víctor Smith Agreda junto a Rudolf Diepen sobre el desarrollo del sistema hipotálamo-hipofisario.

PALABRAS CLAVE: embriología, neuroanatomía, España, franquismo, siglo XX.

KEY WORDS: embryology, neuroanatomy, Spain, Francoism, 20th century. 


\section{Introducción}

Multitud de trabajos recientes han revelado que el proceso de depuración universitaria ${ }^{1}$ y la creación del CSIC $^{2}$ son los dos procesos fundamentales para entender la configuración final de lo que se ha dado en llamar la «ciencia oficial» del régimen franquista. Sin embargo, la historiografía científica aún no ha valorado con precisión este proceso en las diferentes parcelas del saber. En lo que respecta a las ciencias morfológicas, el gran desarrollo de los estudios sobre la escuela histológica española ha hecho sombra en cierta manera a otras especialidades, como la anatomía o la embriología, a las que se les ha prestado escasa atención hasta el momento. La bibliografía que nos ha llegado sobre el asunto se reduce apenas a unos cuantos trabajos biográficos sobre las personalidades más influyentes de la época.

Para estudiar el devenir de la embriología en particular - objeto de este trabajo - hay que entender primero que, durante toda la edad contemporánea, esta disciplina fue pasando de mano en mano entre naturalistas, histólogos, anatomistas y clínicos, sin una adscripción fija. En nuestro país, el único laboratorio que la cultivaba a finales del siglo XIX era el de Cajal. No obstante, para aquél, los embriones no eran más que la base de su «método ontogénico», gracias al cual consiguió describir histológicamente las complejas estructuras del encéfalo adulto merced al estudio de las correspondientes estructuras embrionarias, mucho más asequibles morfológicamente ${ }^{3}$.

1. Son muchos los trabajos sobre el proceso de depuración. Uno de los más completos es el de Claret Miranda, Jaume. La repressió franquista a la universitat espanyola. Universitat Pompeu Fabra i Institut Universitari d'Història Jaume Vicens i Vives, [tesis en internet]; 2004 [citado 25 May 2011]. Disponible en: http://hdl.handle.net/10803/7463, que dio lugar a una publicación en castellano en papel dos años después: Claret Miranda, Jaume. El atroz desmoche. La destrucción de la Universidad española por el franquismo, 1936-1945. Madrid: Crítica; 2006.

2. Sobre el primer CSIC véase: Santesmases, María Jesús; Muñoz, Emilio. Las primeras décadas del Centro Superior de Investigaciones Científicas. Una introducción a la política científica del régimen franquista. Boletín de la Institución Libre de Enseñanza. 1993; 16: 73-94; Urquijo Goitia, José Ramón. Ruptura y creación. Primeros años. In: Puig-Samper, Miguel Ángel, ed. Tiempos de investigación. JAE-CSIC cien años de ciencia en España. Madrid: CSIC; 2007, p. 259-267 y Malet, Antoni, Las primeras décadas del CSIC: investigación y ciencia para el Franquismo. In: Romero de Pablos, Ana; Santesmases, María Jesús, eds. Cien años de política científica en España. Bilbao: Fundación BBVA; 2008, p. 211-256.

3. Puede que el mejor acercamiento a la figura de Cajal sea el de López Piñero, José María. Santiago Ramón y Cajal. Valencia: Publicacions de la Universitat de València y Editorial Universidad de Granada; 2006. Para un estudio más concreto de sus aportaciones a la embriología véase 
Durante las primeras décadas del siglo XX, algunos naturalistas y zoólogos se interesaron por la embriología. De ellos, sólo terminó especializándose en esta disciplina el jesuita Jaime Pujiula (1869-1958) ${ }^{4}$. Su principal objetivo - desmontar la ley biogenética fundamental de Haeckel- le llevó a cultivar preferentemente la embriología comparada y a convertir su Institut en el único centro del país dedicado a la materia.

Conforme fueron pasando los años, los naturalistas fueron perdiendo el interés por la embriología y los médicos ocuparon su nicho. La gran cantidad de líneas de investigación que tenían en marcha los histólogos, que eran en principio los llamados a su cultivo, hizo que terminara convirtiéndose en propiedad casi exclusiva de los anatomistas. Desde hacía décadas tenían cerrada la anatomía macroscópica y buscaban ávidamente nuevos campos de investigación para seguir justificando su amplia presencia en los currículos docentes médicos. Por todo ello, para reconstruir la historia de la embriología española del siglo XX es necesario prestar una especial atención a la historia de estos anatomistas.

Tras la Guerra Civil, se comenzó a fraguar en Cádiz una importante escuela anatómica alrededor de la figura de Francisco Orts Llorca (19051993). Por razones que aún permanecen ocultas ${ }^{5}$, a pesar de una clara vinculación a círculos republicanos, el joven profesor había conseguido sobrevivir a la depuración política y había sido repuesto en su cátedra ${ }^{6}$. Formado en los más importantes centros de Francia y Austria, se convirtió en el introductor de la moderna embriología experimental en España ${ }^{7}$ a

Baratas Díaz, Alfredo. La obra neuro-embriológica de Santiago Ramón y Cajal. Dynamis. 1997; 17, p. 259-279 y Rodríguez Quiroga, Alfredo. Sobre las investigaciones neuro-embriológicas cajalianas: la correspondencia entre Santiago Ramón y Cajal y Wilhelm His. Cronos: Cuadernos Valencianos de Historia de la Medicina y de la Ciencia. 2000; 3 (1): 183-200.

4. Durfort i Coll, Mercé. Jaime Pujiula i Dilmé, SJ. La morfología microscópica. In: Camarasa, J. M. y Roca Rosell, A., dirs. Ciència i tècnica als Països Catalans. Una aproximació biogràfica als darrers 150 anys. Barcelona: Fundació Catalana per a la Recerca; 1995, p. 829-858.

5. En el Archivo General de la Administración (AGA) se conserva el expediente de depuración de Orts Llorca (1937-1977) bajo la signatura IDD (5).001.028, caja 21/20531, exp. 31023-195. No obstante, no será accesible hasta que no hayan transcurrido veinticinco años de su fallecimiento. Su desclasificación, prevista en principio para 2018, probablemente arrojará luz sobre este misterio.

6. Sólo sufrió un descenso en el escalafón de catedráticos. Giral, Francisco. Ciencia española en el exilio (1939-1989). El exilio de los científicos españoles. Anthropos. 1994: 59.

7. Arechaga, Juan; Jiménez-Collado, Juan; Ruano Gil, Domingo. A glance at Spanish embryology and teratology during the XX century through the academic life of Francisco Orts Llorca (1905-1993). International Journal of Developmental Biology. 2009; 53: 1165-1177. 
pesar de que el Régimen nunca apoyó económicamente la investigación de su valiosa escuela.

Y es que, la actividad de los morfólogos españoles durante la posguerra, así como la del resto de científicos del país, se vio profundamente influida por el franquismo como sistema político e ideológico. Durante los primeros años de la Dictadura, la selección del personal científico del nuevo CSIC por parte de las autoridades, y la depuración política en las aulas universitarias, significaron un verdadero tamiz por el que todo el personal investigador del país se vio obligado a pasar. Como consecuencia, y en el grado en que le fue posible al nuevo estado, la «élite académica» quedó dibujada a la medida de su programa político.

Mientras la embriología de Orts maduraba, nacía en Granada otra poderosa escuela anatómica en torno a José Escolar García. A diferencia de su rival, el nuevo catedrático recibió el apoyo incondicional del Régimen a través del CSIC de Albareda. La influencia de ambos en el desarrollo de la anatomía española durante la Dictadura fue abrumadora. A mediados de los años 70, casi el 60\% de las cátedras de anatomía del país estaban regidas por algún miembro de estas dos escuelas. Es más, el cultivo de la embriología se concentró casi exclusivamente en ambos grupos. Con alguna modestísima excepción, los anatomistas ajenos a ellos no se acercaron a las ciencias del desarrollo hasta los años finales de la Dictadura. Por lo tanto, para entender el desarrollo de la especialidad durante el primer Franquismo, resulta indispensable analizar los conceptos teóricos y la producción científica de ambas escuelas.

En el presente trabajo nos proponemos analizar los estudios embriológicos de la escuela anatómica de José Escolar durante los años 40 y 50. De este modo demostraremos si existió un paralelismo con la política científica general del CSIC descrita en trabajos previos y aclararemos si este sistema marcó alguna diferencia esencial con la escuela rival.

\section{José Escolar: creador y organizador de una escuela anatómica}

José Juan Ramón Escolar García (1913-1998) ${ }^{8}$ nació en Vallelado, al noroeste de la provincia de Segovia, aunque muy pronto su familia abandonó aque-

8. Para reconstruir su biografía científica de Escolar se han de consultar fondos del Archivo General de la Administración (AGA): Título de universidad de José Escolar García. Localizado en: AGA, 
lla población. El mismo año de su nacimiento, su padre, Tiburcio Escolar Cantalejo, fue nombrado auxiliar de clases prácticas en la Escuela de Veterinaria de Zaragoza ${ }^{9}$ y, a pesar de algún intento por regresar a Castilla ${ }^{10}$, los Escolar terminaron trasladándose definitivamente a la capital aragonesa, donde José cursó las licenciaturas de Medicina y Veterinaria.

Durante su etapa como estudiante descubrió su interés por la anatomía. En 1932 se incorporó como alumno interno pensionado en la cátedra de Gumersindo Sánchez Guisande (1894-1976) y, una vez graduado, continuó allí como auxiliar temporal. Junto a aquél, Escolar tuvo la oportunidad de conocer una anatomía muy dinámica, gegenbauriana, integradora de la ontogenia y de la filogenia como medios de abordaje de la forma.

Apenas llevaba un año en el puesto cuando consiguió una pensión para estudiar el sistema linfático en el laboratorio de Rouvière en París. Sin embargo, el estallido de la Guerra Civil le obligó a regresar a Zaragoza. Como docente, se militarizó en el bando nacional y como clínico comenzó a servir en el hospital de sangre instalado en el Colegio de El Salvador.

Tras la Guerra, el panorama de la anatomía en la Universidad de Zaragoza había cambiado por completo. La relación de Guisande con la política republicana le había costado su cátedra y había sido sustituido por José Conde Andreu (1895-1958), el hasta entonces catedrático de anatomía de Cádiz. Se había trasladado en el 36 a Zaragoza, su ciudad natal, y durante los años que duró la guerra había sido adscrito a su facultad. Cuando terminó el conflicto, con Guisande fuera de juego, depurado y exiliado, no le resultó difícil hacerse con la cátedra. Para la morfología zaragozana, aquel cambio

32/15265, exp. 91121-40; José Escolar García. Personal Docente. Localizado en: AGA, 32/15190; exp. 8802-98; Expediente de concurso de traslado de Anatomía descriptiva y Topográfica y Técnica Anatómica (1.०) de la Facultad de Medicina de la Universidad de Zaragoza, 1959. Localizado en: AGA, 32/18708, exp. 15358, así como y el expediente de becario en el Archivo de la Fundación Juan March (AFJM. Ayudas para el extranjero. Grupo IV, 1959). Son también de utilidad la necrológica de Vera Gil, Rafael. Profesor José Escolar García. Archivos de la Facultad de Medicina de Zaragoza; 1998, 2, p. 77-78 y la semblanza que le dedicó Gutiérrez Galdó en su historia de la Real Academia de Medicina y Cirugía de Granada: Gutiérrez Galdó, José. Real Academia de Medicina y Cirugía de Granada. Académicos numerarios que fueron. Granada: Ediciones Díaz de Santos; 2003, p. 159-162.

9. Gómez Piquer, J.; Pérez García, J. M. Crónica de 150 años de estudios veterinarios en Aragón (1847-1997). Zaragoza: Institución Fernando el Católico; 2000, p. 142.

10. Sabemos, por ejemplo, que en 1915 opositó a cátedra en la Escuela veterinaria de León. Así consta en: Revista de Higiene y Sanidad Veterinaria. 1915; 5 (9): 802. 
significó la desaparición del dinamismo ontofilogenético y su sustitución por una anatomía más clásica, fundamentalmente quirúrgica y aplicativa.

En este contexto, Escolar y Luis Jiménez González (n. 1912) - el otro auxiliar de la cátedra-, comenzaron a buscar alternativas de formación fuera de Zaragoza. Ambos eran ya doctores en 1940 y en aquel punto de su carrera necesitaban ante todo consolidar su currículum investigador. La situación de aislamiento del país ponía muy difícil las salidas al extranjero, así que se procuraron sendas becas del CSIC para desarrollar una estancia en un laboratorio nacional. Eligieron como destino Valencia, donde el Consejo había creado una sección del Instituto Cajal alrededor de Juan José Barcia Goyanes (1901-2003), que, precisamente, había sido condiscípulo de Guisande en Santiago.

Emulando aquellos centros multidisciplinares de investigación neural que se habían desarrollado en Alemania ya a finales del ochocientos, en la cátedra de Barcia, anatomistas, neurólogos, neuropsiquiatras y neurocirujanos trabajaban al unísono con el sistema nervioso central como objeto de estudio común ${ }^{11}$. La morfología de Barcia era muy novedosa y se basaba en la asunción de la visión funcional de la anatomía alemana ${ }^{12}$. Esta nueva óptica, acuñada en esencia por Hermann Braus (1868-1924), daba un gran valor a la filogénesis para la concepción de la forma, buscaba la causalidad de ésta a través de la experimentación y reordenaba el cuerpo humano en sistemas morfofuncionales ${ }^{13}$. A partir de las tesis de Braus y de los trabajos de otros científicos alemanes y austríacos como Clara, Benninghoff o Heidenhain, Barcia creó su propia concepción anatómica. Teñida de un personal e intenso cariz teleológico, su morfología quedó articulada alrededor de tres axiomas que marcarían profundamente la anatomía de Escolar: (1) La forma como finalidad, (2) la forma como base de la función y (3) la forma como función.

11. Sobre el laboratorio véase Barcia Salorio, Demetrio. Prof. Juan José Barcia Goyanes (1901-2003). In memoriam. Psiquiatría.com. [Citada 12 May 2009]. Disponible en: http://www.psiquiatria. com.es/ilustres/GOYANES.pdf.

12. El núcleo de esta nueva concepción lo encontramos en su discurso de recepción en la Real Academia de Medicina de Valencia, que leyó el 9 de marzo de 1949. Se puede encontrar trascrito en línea: Barcia Goyanes, Juan José. Las orientaciones actuales de la morfología. [Citada 12 Ene 2010]. Disponible en: http://www.ramcv.com/Discursos/ Dr.Barcia-Goyanes.pdf.

13. Véase Nyhart, Lynn K. Learning from history: Morphology's challenges in Germany ca. 1900. Journal of Morphology. 2002; 252 (1): 2-14. 
Por recomendación del catedrático, Escolar comenzó a estudiar la morfogénesis de los plexos coroideos. Los conocimientos prácticos de Barcia en embriología eran realmente muy limitados y en su laboratorio se estaban desarrollando por aquellos años las primeras experiencias en este campo. No obstante, sus contactos paliaron en cierto modo el problema. No hay que pasar por alto que la otra cátedra valenciana de anatomía estaba regida por Rafael Alcalá Santaella (1896-1959), que se había formado inicialmente con Pujiula en Barcelona y, gracias a sendas pensiones de la JAE, había conocido la embriología que se estaba haciendo en los mejores laboratorios europeos del momento. Además, la sección había establecido lazos con el laboratorio del jesuita, lo que permitió incluso que alguno de los becarios del CSIC trabajaran en él.

La modesta embrioteca de Alcalá Santaella fue básica para estos primitivos trabajos y la influencia de la embriología de Pujiula en el laboratorio valenciano se puede detectar sobre todo en la metodología, reducida a una descriptiva pura. Escolar, por el contrario, salió de esa línea aplicando el método de Born desde sus primeras investigaciones ${ }^{14}$.

Descrito por Gustav Born (1851-1900) en 1876, la técnica -bautizada en su idioma original como Plattenmodelliermethode- gozaba ya por entonces de una enorme difusión mundial. Consistía en la proyección a escala sobre unas planchas de cera de los cortes histológicos seriados de la pieza a reconstruir que, una vez apilados, ofrecían al investigador un modelo tridimensional a escala del embrión en bulto redondo. A pesar de su simpleza, había dado grandes frutos a la comunidad científica internacional. En España, sin embargo, no había terminado de cuajar, aunque algunos científicos la habían conocido en Suiza en los años de la Gran Guerra gracias a las pensiones de la Junta para ampliación de Estudios ${ }^{15}$.

14. Escolar, José. Fundamentos y aportaciones sobre la morfogenia del trigémino. Anales Españoles de Odonto-estomatología. 1943; 2: 197-200 y Escolar, José. Nota previa al estudio del desarrollo de los plexos coroideos. Archivo Español de Morfología. 1944; 4 (9): 3-21.

15. Nos referimos a Emilio Ardévol Miralles, Francisco Carreras Verdaguer, Joaquim Trías Pujol y Cosme Valdovinos García, que visitaron el laboratorio de Auguste Eternod (1854-1932) en Ginebra durante la Primera Guerra Mundial y aprendieron la técnica. Raúl Velasco Morgado. Importando una ciencia médica básica. los viajes para investigaciones embriológicas de los pensionados de la JAE. In: Porras Gallo, M. Isabel; Gutiérrez Rodilla, Bertha; Ayarzagüena Sanz, Mariano; De las Heras Salord, Jaime (eds.). Transmisión del conocimiento médico e internacionalización de las prácticas sanitarias: una reflexión histórica. XV Congreso de la Sociedad Española de Historia de la Medicina. Ciudad Real: SEHM, Facultad de Medicina de Ciudad Real y Universidad de Castilla-La Mancha; 2011, p. 109. 
Tras la estancia en Valencia, los auxiliares de Zaragoza regresaron a su universidad de origen y poco después Escolar se trasladó a Granada. Se le había nombrado responsable de cátedra y comenzó a preparar la oposición. Para cubrir gastos, le fue concedida una de las veintitrés becas nacionales «José Miguel Guitarte» ${ }^{16}$, dirigidas a licenciados que estuvieran opositando a cátedra y que tuvieran limitados recursos económicos. Habían sido convocadas por primera vez aquel año y dependían de la Sección Nacional de Graduados del Sindicato Español Universitario (SEU), una agrupación altamente politizada, nacida en el seno de la Falange, y que, establecida por el Régimen de Franco como la única agrupación estudiantil legal, mantenía el monopolio de la concesión de becas.

Así, gracias al apoyo de dos poderosas instituciones - CSIC y SEU-, manejadas por dos fuerzas que pugnaban por el poder en aquella etapa de la dictadura -Opus Dei y Falange respectivamente-, Escolar consiguió el currículum necesario para acceder a la cátedra en 1945.

\section{Los comienzos}

Establecido en Granada, Escolar se encontró con el reto de desplegar su propio itinerario como docente e investigador en aquel contexto de posguerra. En pocos meses, su estilo pedagógico marcó una clara diferencia con sus predecesores. Como recordó uno de los alumnos de su primer curso académico, con él cada estructura anatómica comenzó a describirse: «con sus brillantes dibujos de color en la pizarra [...] por su origen ontogénico $\mathrm{y}$, sobre todo, por su función» ${ }^{17}$. Pero, para llevar a cabo una revolución idéntica en el ámbito de la investigación, los medios con los que contaba eran más que limitados.

No obstante, y a pesar de la situación del país, el Instituto Anatómico de Granada se acababa de construir de nueva planta. El edificio había sido proyectado personalmente por el otro catedrático de anatomía, Miguel Guirao Gea, y había sido inaugurado el 2 de julio de 1944. En aquellos años, los nuevos locales fueron calificados como «el modelo más perfecto

16. Declaraciones del Secretario General de la Sección de Graduados en el duodécimo aniversario de la fundación del SEU. ABC. 21 Nov 1945: 21.

17. Cruz Hernández, Manuel. José Escolar: clásico y genio de la anatomía. Seminario Médico. 2003; 55 (2): 113. 
y completo de los de su clase en España y equiparable a los mejores del mundo» ${ }^{18}$.

Sin embargo, el mantenimiento de un laboratorio de morfología competitivo a nivel internacional era muy costoso y la universidad española no se lo podía permitir en aquel momento. Ante esta crítica situación financiera, Escolar mostró una gran capacidad de adaptación. Reinoso, uno de los primeros alumnos internos, da cuenta del ingenio de su maestro:

«[Vi a Don José] desde construir una ampliadora o un aparato de microfotografías caseros por no disponer de presupuesto para ellos, hasta hacerse un gran micrótomo para cerebros humanos en un taller de reparación de automóviles (...)

(...) disponíamos sólo de un microscopio, que además era el utilizado en las microfotografías, por lo que había que repartir el trabajo durante las 24 horas del día y los siete días de la semana» ${ }^{19}$.

Decidido a proseguir la línea de embriología descriptiva que había comenzado en Valencia, el nuevo catedrático fue iniciando a sus discípulos en lo más básico de esta ciencia. Otro de sus discípulos recordaba:

«Cuando llegó, Escolar [...] tenía alguna, poca, formación embriológica, lo que ocurre es que era muy creativo y de poco sacaba mucho: Empezamos haciendo una embriología puramente morfológica y muy intuitiva" (incluir cortar, teñir y mirar, no sé si embriones de rana, y poco más ${ }^{20}$.

Aprendimos lo básico para desenvolverse en la investigación embriológica, es decir, todas las técnicas de inclusión en parafina y celoidina, reconstrucciones de Born, embriología teórica descriptiva, etc.» ${ }^{21}$

18. Olagüe de Ros, Guillermo. Sobre sólida roca fundada: Ciento veinte años de labor docente, asistencial e investigadora en la Facultad de Medicina de Granada (1857-1976). Granada: Universidad de Granada; 2001, p. 206. Guirao nos dejó una descripción muy detallada del instituto en: Guirao Gea, Miguel. El nuevo Instituto Anatómico de la Facultad de Medicina de Granada (España). Arquivo de Anatomia e Antropologia. 1947; 24: 477-480.

19. Reinoso Suárez, Fernando. Algunas notas sobre mi vida. [Boletín de la] Sociedad Española de Neurociencias. 2003; 13: 14-15.

20. Guirao Pérez, Miguel. Correo electrónico al autor. Granada, 19 de diciembre de 2009. Localizado en: Archivo del autor, sin catalogar.

21. Guirao Pérez, Miguel. Currículum vitae (exclusivamente de la labor embriológica) [1959]. Localizado en: AFJM. Ayudas para la investigación en España. Grupo IV. Expediente de Miguel Guirao Pérez. fol. 35-36. 
Así era, el equipo siguió utilizando el clásico método de Born para las reconstrucciones tridimensionales, pero, de forma paralela, fueron incorporando otras técnicas descriptivas, como la estereofotométrica de Sobotta-Neumayer o la planimétrica de His. En ellas, Escolar encontró el cariz holístico que quería dar a su anatomía y a su embriología, una óptica que, a su entender, no le podían ofrecer las técnicas de la embriología experimental, que le resultaban «parciales». Esta tendencia, que marcó la diferencia con la escuela de Orts Llorca, quedó claramente reafirmada en un texto de 1947:

«Comprendemos que nuestros procedimientos de estudio son los mismos que emplearon aquellos investigadores de tiempo atrás. Otros procederes diferentes se generalizan hoy día por ser más sencillos. Son procederes dinámicos que buscan la dinámica de la cuestión con bases dinámicas puras, según el criterio de los que los emplean; pero estos resuelven el problema en líneas generales, como corresponde a la unidad individual de nuestro organismo, única realidad. Sin embargo, nadie duda que la expresión vital, y que, en consecuencia, de la forma, no se puede prescindir, como tampoco de aquellos procedimientos laboriosos de investigación, máxime si sus resultados se someten a consideraciones funcionales dependientes de la unidad individual ${ }^{22}$.

Los trabajos de Escolar durante aquel primer año se concretaron en un artículo sobre el desarrollo del seno venoso que apareció en Archivo Español de Morfología ${ }^{23}$. Esta publicación, editada por la cátedra de Barcia, se convirtió en el principal medio de comunicación del equipo granadino durante esta primera etapa.

La protección de Escolar por parte del CSIC es un hecho constatable desde estos años. Nada más llegar a Granada, el catedrático fue nombrado profesor agregado del Cajal y fue beneficiado con una nueva beca de viaje. A partir de septiembre de 1946 y durante ocho meses, su lugar de trabajo fue el Department of Anatomy de la Northwestern University Medical School, en Chicago, un centro que dirigía el prestigioso neurocientífico Horace Winchell Magoun (1907-1991). Terminada la Segunda Guerra Mundial, el gobierno buscaba claramente alianzas con los vencedores ${ }^{24}$.

22. Escolar García, José. Aportaciones al desarrollo del seno venoso. Archivo Español de Morfología. 1947; 5 (16): 425

23. Escolar García, n. 22.

24. Delgado Gómez Escalonilla, Lorenzo. Dimensión internacional del CSIC. In: Puig-Samper Mulero, Miguel Ángel, ed. Tiempos de investigación: JAE-CSIC, cien años de ciencia en España. 
La embriología de aquel centro había tenido como exponente más importante a George William Bartelmez (1885-1967), pero cuando llegó Escolar el reputado morfólogo se encontraba en una pausa dentro de sus investigaciones embriológicas y estaba dedicado a la investigación morfológica funcional ${ }^{25}$. De hecho, todo el departamento compartía este interés por la morfofisiología y mantenía una intensa producción en el área neurofuncional.

En este ambiente, el joven científico español descubrió que su embriología estaba relativamente desfasada y quedó deslumbrado ante el potencial investigador de las nuevas técnicas neurofisiológicas. Haciendo a un lado a la embriología, durante toda la visita se centró en adquirir el máximo conocimiento sobre las nuevas técnicas de degeneración walleriana ${ }^{26}$. A su vuelta, comenzó una titánica labor publicista para presentar a la comunidad científica española los resultados obtenidos con los métodos aprendidos en América. Sorprendentemente, a pesar del descomunal impacto de la nueva técnica, el anatomista no abandonó la embriología y durante la segunda mitad de la década siguió preocupado por la neuroembriología ${ }^{27}$.

A la fructífera vivencia de Escolar en el extranjero le siguieron una serie de experiencias similares por parte de todos los miembros de su equipo. A finales de los años 40, Juan Jiménez-Castellanos y Calvo-Rubio (1923-2009), el adjunto a la cátedra, fue el primero en salir al extranjero. Siguiendo los pasos del maestro, viajó hasta Chicago con el claro objetivo de adiestrarse en las técnicas electrofisiológicas.

Natural de Aguilar de la Frontera, una villa de la campiña sur de la provincia de Córdoba, había estudiado Medicina en la Universidad de Granada $y$, al igual que el resto de doctorandos de Escolar, había centrado su tesis en el desarrollo neurovascular, concretamente en el del espacio retroperitoneal. Posteriormente siguió con esta línea embriológica y comenzó una serie

Madrid: CSIC; 2007, p. 272-273 y Canales Serrano, Antonio Francisco. La política científica de posguerra. In: Gómez, Amparo; Canales Serrano, Antonio Francisco. Ciencia y Fascismo. Barcelona: Laertes; 2009, p. 127.

25. Bodian, David. George William Bartelmez 1885-1967. Washington D.C.: National Academy of Sciences; 1973.

26. CSIC, Memoria de la Secretaría General, Año 1946-1947. Madrid: CSIC; 1948, p. 389.

27. Escolar, José. Sobre la formación del núcleo rojo. Archivo español de Morfología. 1949; 22: 123-135; Escolar, José. Sobre la formación de la oliva bulbar. Archivo español de Morfología. 1949; 25: 395-406 y Escolar, José. Aportaciones al estudio del núcleo «centrum medianum de Louis». Un análisis del tálamo en desarrollo. Actualidad Médica. 1949; 35 (300): 689-693. 
de trabajos en los que se acercó a la morfogenia de los sistemas arteriales cerebrales $^{28}$ y a otros temas neuroembriológicos, fundamentalmente al desarrollo del córtex y de los núcleos caudado y lentiforme ${ }^{29}$.

En el curso 1948-1949 y durante nueve meses, tuvo la ocasión de practicar las técnicas electrofisiológicas con el propio Magoun, con Krieg y con Niemer. Sin embargo, a diferencia de Escolar, este campo no monopolizó su estancia en EEUU y decidió compaginarlo con algunas investigaciones embriológicas. Para ello recibió la tutela de Leslie Brainerd Arey (1891$1988)^{30}$, un histólogo del departamento que había mostrado un gran interés por la morfogenia; de hecho, era el autor de un manual de embriología y técnica embriológica que había contado con gran aceptación en EEUU y que, cuando Jiménez-Castellanos llegó a EEUU, iba ya por su quinta edición.

Junto a Brainerd siguió con el tema de su tesis y profundizó en el desarrollo de las estructuras neurovasculares retroperitoneales. Con los resultados de estas investigaciones publicó una serie de artículos que aparecieron en tres revistas: la del departamento americano, la portuguesa Arquivo de Anatomia y el Boletín de la Universidad de Granada ${ }^{31}$. Aunque el impacto de estos trabajos fue prácticamente nulo en comparación con el éxito de sus publicaciones sobre estereotaxis, son un claro síntoma de la diversidad de intereses de Jiménez-Castellanos durante esta etapa de su vida.

A su regreso, sus objetivos se diversificaron aún más. Durante el resto de la década compaginó la producción de exitosos estudios sobre estereotaxia y degeneración walleriana con el cultivo de la anatomía macroscópica y la embriología, aspectos íntimamente ligados en la morfología de Escolar. Por

28. Jiménez-Castellanos, Juan. Aportaciones al estudio ontogénico de la circulación basilar del encéfalo. Actualidad Médica. 1947; 33: 203-225; Jiménez-Castellanos, Juan. Ontogénesis arterial intraencefálica. Actualidad Médica. 1948; 34 (281): 259-264 y Jiménez-Castellanos, Juan. El sinus cavernosus y sus relaciones embriológicas con los órganos vecinos. Archivo Español de Morfología. 1949; 25: 453-482.

29. Jiménez-Castellanos, Juan. Modificaciones tintoriales somáticas en el desarrollo neuronal. Boletín de la Universidad de Granada. 1947; 21; Jiménez-Castellanos, Juan. Prioridad evolutiva de la cortex cerebri y de los nuclei caudatus y lentiformis. Arquivo de Anatomia e Antropologia. 1948; 26: 339-359.

30. Así lo afirma en: Jiménez-Castellanos y Calvo Rubio, Juan. The phases of development of the paraganglionar suprarrenal systems. Arquivo de Anatomia e Antropologia. 1947; 27: 219-293.

31. Jiménez-Castellanos y Calvo-Rubio, n. 29; Jiménez-Castellanos y Calvo-Rubio. The morphogenesis of the systems of yuxta-aortics tissues in human embryos. Quarterly Bulletin Northwestern University Medical School 1949; 23 (4): 428-431; Jiménez-Castellanos y Calvo-Rubio, Juan. Sobre el desarrollo de los sistemas arterial y cromafín retroperitoneales. Boletín de la Universidad de Granada. 1950; 22 (88): 151-167. 
aquellos años, el catedrático estaba construyendo su particular anatomía, y el concepto de sistema neuromuscular estaba madurando como nueva unidad anatómica. Con una clara influencia del funcionalismo alemán y el teleologicismo de Barcia, se entiende por sistema neuromuscular de Escolar «el complejo anatomo-funcional, diferenciado por la teleología embrionaria, constituido por un grupo de músculos y un sistema de nervios que se encargan de inervarlos para que puedan realizar una determinada y específica función» ${ }^{32}$. La embriología era, pues, indispensable para definir estas estructuras morfo-funcionales, así que, para completar el proyecto, todos los investigadores de la cátedra se pusieron manos a la obra con el fin de aportar los datos morfogénicos necesarios ${ }^{33}$.

Además de involucrarse en esta línea ${ }^{34}$, Jiménez-Castellanos siguió dedicando algunos esfuerzos a la neuroembriología hasta finales de los años $40^{35}$. Durante la década siguiente, la embriología fue perdiendo peso en su producción y terminó desapareciendo. En 1955 ganó la cátedra de Salamanca y sólo un año después se trasladó a la de Sevilla. Durante su breve estancia en Salamanca, se le llegó a considerar oficialmente un apéndice del equipo de Granada especializado en morfogenia y técnica embriológica ${ }^{36}$, pero apenas publicó cinco trabajos de embriología después de $1950^{37}$. Sus objetivos eran otros y terminó decantándose por una subespecialidad mucho

32. Definición recogida de Smith Agreda, Víctor; Ferres-Torres, Elvira. Fascias: principios de anatomofisio-patología. Barcelona: Paidotribo; 2004, p. 370.

33. El proyecto de la descripción de los sistemas neuromusculares se extendió durante años y quedó plasmado en su conocido tratado: Escolar, José. Anatomía humana funcional y aplicativa. Zaragoza: Imprenta Heraldo de Aragón; 1965.

34. Jiménez-Castellanos, Juan. Análisis morfogenético de ciertas variedades del dispositivo muscular serrato angular. Actualidad Médica. 1950; 36 (304): 432-466.

35. Jiménez-Castellanos y Calvo-Rubio, Juan. Prioridad evolutiva del pallium cerebri y de los nuclei caudatus y lentiformis. Arquivo de Anatomia e Antropologia. 1949; 26: 339-359; JiménezCastellanos, Juan, El sinus cavernosus y sus relaciones embriológicas con los órganos vecinos. Archivo Español de Morfología. 1949; 8 (25): 453-482; Jiménez-Castellanos, Juan. Morfogénesis del substrato de drenaje cefalorraquídeo referido a las primeras fases del desarrollo. La Prensa Médica Argentina. 1950; 36 (33): 1901-1908.

36. Consejo Superior de Investigaciones Científicas. Secretaría General, Memoria 1955-1957. Madrid: CSIC, 1959, p. 438-439.

37. Jiménez-Castellanos, Juan. Contribución al estudio morfogenético de la musculatura retrosomática. Memorias de la Real Academia de Ciencias Exactas, Físicas y Naturales de Madrid. Serie de Ciencias Naturales. 1953; 17 (3): 5-55; Jiménez-Castellanos, Juan. Aportaciones a la morfología del dispositivo neuromuscular «subscapularis». Acta Salmanticensia. Medicina, Vol. I. p. 7-19; Jiménez-Castellanos, Juan. Estudio reconstructivo y estereofotométrico de un embrión de 10 milímetros. Revista de la Real Academia de Ciencias Exactas, Físicas y Naturales de Madrid. 
más rentable: la aplicación de la neuroanatomía funcional a la neurología y a la neurocirugía.

\section{La «Sección de Anatomía» del Instituto Cajal}

En la Universidad de Granada, la creación de secciones periféricas del CSIC sobre determinadas cátedras fue un fenómeno verdaderamente notable. Sólo contabilizando las que dependieron del Cajal encontramos hasta tres ${ }^{38}$. En 1949, la cátedra de Escolar se convirtió en la Sección de Anatomía del Instituto. Desde entonces, la influencia de Escolar en el CSIC sufrió un ascenso vertiginoso hasta llegar a ser nombrado en 1955 consejero del Patronato Santiago Ramón y Cajal, uno de los más altos cargos de la sección biomédica.

La nueva sección se propuso como objetivo resolver a través de la embriología el «significado de la forma, estructuras y organizaciones, para lo cual la disección del adulto y otras macrotecnias planteaban el problema morfogénico» ${ }^{39}$. A pesar de esta declaración de intenciones, un repaso a las publicaciones y tesis defendidas en la cátedra en el quinquenio 1945-1951 pone de manifiesto que los estudios con las nuevas técnicas de degeneración walleriana fueron ganando posiciones hasta ponerse a finales de la década a la altura de las investigaciones embriológicas. Durante este período se defendieron hasta 18 tesis con temas embriológicos, la mayoría de ellas centradas en el desarrollo embrionario de diferentes sistemas vásculo-nerviosos.

En estos años el equipo terminó por consolidarse. José Soler Viñolo (n. 1923) y Fernando Reinoso Suárez (n. 1927) se unieron como personal fijo y Miguel Guirao Pérez (1924-2010), hijo de Guirao Gea y aún estudiante de Medicina, se incorporó desde 1947 como becario del Consejo. Además de las obligadas experiencias con la estereotaxis, Viñolo y Guirao colaboraron en la línea embriológica de la cátedra y aportaron a la anatomía de los sis-

1955; 49 (3): 223-260. Jiménez-Castellanos, Juan. El sistema arterial axilaris: consideraciones morfológicas y ontogénicas. Madrid: Consejo Superior de Investigaciones Científicas, 1960.

38. Olagüe de Ros, n. 17, p. 198-201.

39. Consejo Superior de Investigaciones Científicas. Memoria, año 1949. Madrid: CSIC; 1949, p. 205. 
temas neuromusculares una pequeña serie de trabajos sobre la morfogenia de los diferentes plexos nerviosos ${ }^{40}$.

Desde entonces, las aportaciones de Viñolo a la embriología fueron muy puntuales y terminó por dedicarse casi exclusivamente a la estereotaxia. Guirao, por el contrario, siguió con sus investigaciones embriológicas ${ }^{41}$. Quizá por la libertad que le ofrecía ser el hijo del poderoso Guirao Gea, se convirtió en el único nexo de las dos grandes escuelas anatómicas españolas. En vez de acercarse a Alemania - destino preferido por los escolarianos en aquellos años-, decidió viajar hasta Suecia financiado por el CSIC y aprender embriología al lado de Edvard Holmdahl (1887-1956). Además, a su vuelta, realizó una serie de estancias en el laboratorio de Orts en Madrid, con quien estableció unos vínculos que modificaron en gran parte su embriología, que quedó muy distanciada de la de sus condiscípulos ${ }^{42}$.

\section{La embriología en la visión alemana de la neuroanatomía}

El contexto sociopolítico de los años 50 favoreció que el CSIC desplegara un programa de relaciones internacionales en el que los lazos con la ciencia germana fueron especialmente favorecidos ${ }^{43}$. Durante toda la década

40. Escolar García, José. Aportaciones al desarrollo y sistematización del plexo solar. Boletín de la Universidad de Granada. 1945; 80: 409-422; Campos Martínez, Francisco. Sobre el desarrollo del plexo nervioso hipogástrico. Archivo Español de Morfología. 1947; 5 (16): 449-474; Guirao Pérez, Miguel. Sobre el desarrollo del plexo lumbar. Archivo Español de Morfología. 1948; 6 (17): 3-36 y Soler Viñolo, José. Sobre el desarrollo del plexo sacro. Archivo Español de Morfología. 1948; 6 (17): 37-65. Soler Viñolo, José. Morfología de los tubérculos cuadrigéminos en las últimas fases del desarrollo embrionario. Archivo Español de Morfología. 1950; 8 (27): 23-29 y Soler Viñolo, José. Aportaciones al desarrollo del tectoencéfalo. Archivo Español de Morfología. 1950; 8 (28): 109-151.

41. Guirao Pérez, Miguel. Evolución de la sustancia gris yuxtacalámica. Archivo Español de Morfología. 1949; 23: 257-280 y Guirao Pérez, Miguel. Morfología de los núcleos yuxtacalámicos en las últimas fases del desarrollo. Archivo Español de Morfología. 1949; 26: 577-609; Guirao Pérez, Miguel. Ontogénesis arterial del miembro inferior. Arquivo de Anatomia e Antropologia. 1950; 5 (27); Guirao Pérez, Miguel. La columna motora branquiógenea. Morfología y evolución, Arquivo de Anatomia e Antropologia. 1952; 27: 229-240

42. Velasco Morgado, Raúl. Un proyecto personal al margen de las escuelas hegemónicas: el instituto «Federico Olóriz» de Estudios Anatómicos. Llull. Revista de la Sociedad Española de Historia de las Ciencias y de las Técnicas. 2014; 37 (80): 169-198.

43. Presas i Puig, Albert. La inmediata posguerra y la relación científica y técnica con Alemania. In: Romero de Pablos, Ana; Santesmases; María Jesús, eds. Cien años de política científica en España. Bilbao: Fundación BBVA; 2008, p. 176-178. 
fueron muchos los desplazamientos de Escolar a la República Federal Alemana (RFA). Gracias al esfuerzo económico del CSIC y al suyo propio, el catedrático español consiguió consolidar vínculos con un gran número de científicos de aquel país, sobre todo con Hugo Spatz (1888-1979), el director del Max Planck-Institut für Hirnforschung ${ }^{44}$, el centro especializado en el estudio del cerebro de la red científica estatal alemana.

Spatz era psiquiatra y neuropatólogo, y en estas áreas había destacado en las décadas anteriores ${ }^{45}$; pero la faceta que más marcó su biografía fue su vinculación con el Tercer Reich y el uso que hizo de los resultados de las campañas eugenésicas de los nazis como material de investigación ${ }^{46}$. De hecho, una gran parte de la preciosa colección de cortes encefálicos que atesoraba el centro y que utilizaron los escolarianos en Alemania tenía este macabro origen. Su instituto, localizado en Giessen, dedicaba fundamentalmente sus esfuerzos al cultivo de la floreciente anatomía neuroendocrinológica y a la integración de la anatomía comparada y la embriología en la neuroanatomía, en un concepto haeckeliano que denominaban «visión evolutiva».

La Sección de Anatomía del CSIC y la comunidad anatómica alemana fueron estrechando lazos hasta convertirse en un ejemplo paradigmático de la política germanófila del Consejo. Spatz llegó a ser investido doctor honoris causa por la Universidad de Granada en 1955 y, durante la década, se multiplicaron las estancias de los discípulos de Escolar en la RFA y las visitas de los profesores alemanes a Granada. En 1958, por ejemplo, viajaron a España el propio Spatz, su compañero Rudolf Diepen y científicos de otros laboratorios germanos como Rolf Hassler (1914-1984), del instituto anatómico de Freiburgo.

Durante aquellos años, los intereses científicos de los escolarianos se fueron diversificando y sufrieron en buena medida un proceso de subespecialización. Aunque sin convertirse en compartimentos estancos, algunos se vieron atraídos por la neuroendocrinología, otros siguieron con la línea electrofisiológica y otros abordaron la neuroanatomía desde los puntos de

\footnotetext{
44. Satzinger, Helga. Max-Planck-Institut für Hirnforschung: Berlin-Frankfurt. In: Denkorte. MaxPlanck-Gesellschaft und Kaiser-Wilhelm-Gesellschaft: Brüche, Kontinuitäten, Erinnerungen 1911-2011. Sandstein Kommunikation; 2010, p. 294-301.

45. Bielk, Heinz. Geschichte der Medizinisch-Biologischen Institute Berlin-Buch. Zweite Auflage. Springer: Berlin y Heidelberg; 2002, p. 178-179.

46. Sobre este aspecto en concreto véase Aly, Göt; Chroust, Peter; Pross, Christian. Cleansing the fatherland: Nazi medicine and racial higiene. JHU Press; 1994, p. 147.
} 
vista embriológico y filogenético. Siguiendo nuestros objetivos, nos centraremos aquí en esta última línea de investigación que, durante la década de los 50, abordaron, además de Escolar, Fernando Reinoso y Víctor Smith.

La ciencia alemana proporcionó al grupo nuevas técnicas para las investigaciones embriológicas del grupo. Adoptaron las tinciones de Nissl y de Heidenhain-Wölke para la mielina y conocieron las modificaciones que se habían introducido en el laboratorio de Spatz al método de Born. En vez de la clásica y frágil cera, en Giessen utilizaban un nuevo material plástico, el Schaumstoff, que había sido introducido en la práctica embriológica por un asistente técnico del Institut llamado Höhn.

La actividad de Escolar durante sus múltiples estancias en Alemania giró en torno a la neurosecreción y la aplicación de la visión evolutiva ontofilogénica al problema de la morfología cerebral. En esta última línea, las estructuras sobre las que más trabajó fueron la amígdala y el allocórtex. Para desgranar el desarrollo de éstas, realizó decenas de observaciones sobre cerebros de pequeños mamíferos como la musaraña gris (Crocidura russula) o la tupaya (Tupaia glis) y en individuos de dos especies de primates, el gálago enano (Galagoides demidovii) y el mono Rhesus (Macaca mulatta). Posteriormente, tal y como hacían en el laboratorio alemán, completó aquella primera fase filogenética con una segunda en la que abordó su paralelismo embriológico, una labor que resultaba sencilla gracias a la completa embrioteca que había atesorado Spatz.

En esta serie de estudios, Escolar actualizó su embriología implementando el concepto de citoarquitectura, un término con el que los alemanes se referían a la unidad funcional de ciertas áreas con una disposición histológica particular. Antes de la guerra, el Institut había dedicado buena parte de sus trabajos a la compartimentación de las estructuras cerebrales en orden a esta citoarquitectura. Del caso concreto de la amígdala se había ocupado Brockhaus, que había creado una complicada segmentación de esta estructura y, aunque estadounidense Thomas Johnston había elaborado una subdivisión mucho más sencilla y con una mayor difusión, Escolar adoptó la compleja segmentación alemana y estudió la cronología del desarrollo de cada una de las múltiples secciones en las que Brockhaus había dividido la estructura.

Su hallazgo más importante fue el paralelismo entre el desarrollo filogenético y el embriológico de esta cronología. Utilizando la tinción de Nissl describió cómo las áreas periamigdalinas rostral y lateral y el área prepiriforme tendrían una temprana diferenciación ontofilogenética, en 
oposición a las áreas amigdalinas profundas, periamigdalinas medial y caudal, supraamigdalina y el área entorrinal, que se diferenciarían más tarde. En cuanto a la embriología del allocórtex, sus trabajos mostraron cómo las primeras secciones en aparecer serían la banda diagonal y el tubérculo olfatorio; posteriormente aparecería el área prepiriforme, una región que Escolar vio regresar a la vez que iban desarrollándose el asta de Amón y la región entorrinal.

A finales de la década había recopilado suficientes datos como para publicar varios artículos sobre el tema ${ }^{47}$. La gran mayoría aparecieron en su propia revista, Anales de Anatomía, una publicación que desde 1954 editaban la Universidad de Granada y el CSIC. Este dato parece coincidir $a$ priori con las líneas generales del Consejo en materia publicista, que, según Malet ${ }^{48}$, fomentó durante estos años la autopublicación y volcó gran parte de sus esfuerzos en la creación de personajes de gran reputación local, pero de escaso renombre internacional. El caso de Escolar, sin embargo, no se ajustó del todo a este modelo. Las publicaciones periódicas alemanas de la especialidad fueron también grandes receptoras de sus trabajos, lo que le fue proporcionando un gran impacto internacional, aunque, eso sí, de una forma muy circunscrita a los países del área de influencia germánica.

En 1950 terminó la licenciatura uno de sus alumnos internos, Fernando Reinoso Suárez, un granadino nacido en 1927 en Mecina Bombarón ${ }^{49}$. En 1951 consiguió el título de doctor y se vinculó a la cátedra como ayudante de clases prácticas. Aquel año y el siguiente viajó al Neurophysiologische Abteilung del Max-Planck, en Göttingen, un laboratorio muy especializado

47. Escolar, José. Aportaciones al conocimiento el complejo amigdalino del cerebro humano (consideraciones sobre su arquitectonia, génesis y topografía). Anales de Anatomía. 1953; 2: 5-21; Escolar, José. Desarrollo del complejo amigdalino humano, relacionado con el allocórtex. Anales de Anatomía. 1958; 12, Escolar, José. Aportaciones al desarrollo del allocórtex. Archivos de la Facultad de Medicina de Zaragoza. 1959; 7 (6): 1029-1041; Escolar, José. La distribución allocortical del uncus en relación con su desarrollo embriológico. Trabajos del Instituto Cajal de Investigaciones Biológicas. 1959; 51-52: 57-70; Escolar, José. Aportaciones a la relación entre el allocortex y complejo amigdalino en la escala zoológica, insectívoros primates (Un estudio sobre reconstrucciones de Crocidura russula, Tupaia glis, Gálago demidovii y Macacus rhesus). Anales de Anatomía. 1959; 8 (14): 5-21; Escolar, José. El complejo amigdalino en relación con el allocortex considerado ontogénica y filogénicamente. Anales de Anatomía. 1959. 8: 215-231; Escolar, José. The amygdaloid nucleus complex and its relations to the allocórtex. Verhandlungen der Anatomischen Gesellschaft. 1960: 206-211.

48. Malet, Antoni. n. 2. p. 242.

49. Reinoso Suárez, Fernando. Algunas notas sobre mi vida. Sociedad Española de Neurociencias. 2003; 13: 12-30. 
en neurofisiología y que nada tenía que ver con la morfogénesis. En Granada, sin embargo, había comenzado a colaborar en las líneas de embriología descriptiva del equipo ${ }^{50}$.

Durante toda aquella década, el joven doctor continuó desplazándose casi todos los veranos a Alemania. En 1953, 1954 y 1956 estuvo en el laboratorio de Spatz, y en 1958 en el de Dietrich Stark (1908-2001) en Frankfurt. Ya hemos hablado del interés por la ontogenia del primero, y Stark tampoco estaba alejado de esta visión; manejaba una morfología muy amplia en la que tenían cabida la primatología, la embriología y la neuroanatomía. De este modo, y de forma paralela a sus trabajos sobre vías nerviosas, Reinoso utilizó las embriotecas de ambos centros para dar un apoyo ontogénico a sus investigaciones sobre el diencéfalo.

En 1957 ganó cátedra en Salamanca y allí consiguió «la suficiente infraestructura material y el principio de un grupo científico multidisciplinario que podía ser competitivo internacionalmente ${ }^{51}$. Pero el errar por las geografía universitaria española no había hecho nada más que empezar. En 1960 volvió a Granada para ocupar el puesto de su maestro - que había ganado por concurso de traslado la cátedra de Zaragoza-, y al año siguiente se trasladó a la joven universidad de Navarra, donde encontró cierta estabilidad y pudo trabajar el resto de la década.

Gracias al material embriológico recogido en Giessen y en Frankfurt, durante este período afinó detalles sobre el desarrollo del diencéfalo, sobre todo en lo referente a su relación con el sistema reticular ascendente de activación ${ }^{52}$. Siguiendo con la filosofía anatómica del grupo, la embriología

50. Reinoso Suárez, Fernando. Desarrollo del sistema venoso tributario de los troncos innominados. Anales de Anatomía. 1952; 1: 95-109; Reinoso Suárez, Fernando. Estudio morfológico y embriológico de la arteria carótida externa y sus ramas colaterales. Actualidad Médica. 1954; 40: 657-665. Reinoso Suárez, Fernando. Sobre el conocimiento anatómico y ontogénico de las venas yugulares interna y externa. Anales de Anatomía, 1955; 4: 237-270; Reinoso Suárez, Fernando. Aportaciones al desarrollo de la sustancia negra, zona incerta, cuerpo de Luys y núcleo reticular. Actualidad Médica. 1951; 37: 278-285; Reinoso Suárez, Fernando. La encrucijada retrosomática cérvico-torácica. Anales de Anatomía. 1955; 4: 55-71.

51. Reinoso Suárez. n. 49, p. 19.

52. Reinoso Suárez, Fernando. Sobre las primeras fases del desarrollo de formaciones derivadas de la placa basal del mesencéfalo y diencéfalo. Anales de Anatomía. 1954; 3: 231-245. Reinoso Suárez, Fernando. Desarrollo del subtálamo humano. Anales de Anatomía. 1960; 9: 5-33; Reinoso Suárez, Fernando. El sistema reticular ascendente de activación. Desarrollo de su escalón diencefálico en el hombro y estudio experimental en el gato. Madrid: Publicaciones del CSIC, 1961. 
no era para él más que una pieza en un puzle en el que iba delimitando estructuras neurales de una forma dinámica. La relevancia de sus hallazgos fue tal, que llegó a ser invitado por el propio Giuseppe Moruzzi (1910-1986), codescubridor de la sustancia reticular ascendente, para que expusiera sus resultados en Pisa ${ }^{53}$.

Eran tantos los intereses de Reinoso y tantas las líneas que la neurociencia le brindaba, que decidió centrarse en la investigación electrofisiológica y descartar de su producción científica la embriología y otras áreas que estaban cultivando otros escolarianos. Aun así, cuando en 1969 se trasladó a la Universidad Autónoma de Madrid, no olvidó la importancia que daba su escuela a la embriología y creó una embrioteca para la docencia ${ }^{54}$.

Algo más tarde que Reinoso, en 1955, se incorporó a la cátedra como adjunto el zaragozano Víctor Smith Agreda (n. 1928). Siguiendo el modelo que ya conocemos, se agregó al CSIC y comenzó sus viajes a Alemania. Sus estancias, no obstante, habían comenzado años atrás, en 1952, cuando, gracias a una beca de la Deutsche Akademische Austausdienst, pudo realizar una estancia de diez meses en el Max-Planck. Posteriormente, becado por el Consejo, regresó al mismo departamento en sendas ocasiones (1954 y 1956) y se especializó en morfología neuroendocrinologica.

Estudió la embriología del sistema neurosecretor hipotálamo-hipofisario al lado de Rudolf Diepen y ambos, junto a Engelhardt, describieron el desarrollo embriológico del sistema de comunicación entre ambas estructuras ${ }^{55}$. Además, presentó un trabajo al Congreso Internacional de Anatomía que se celebró en París aquel año, donde defendió que las células del núcleo supraóptico del hipotálamo son embriológicamente neuronas, como las del resto de núcleos «no secretorios» del hipotálamo.

En 1957, con el objetivo de seguir su línea de investigación, decidió volver al Max-Planck y visitar alguno de los laboratorios alemanes que

53. Reinoso Suárez, Fernando. Cajal hoy: la permanencia de un genio. Revista Española de Patología. 2002; 35 (4): 373. Sus descubrimientos quedaron plasmados en un capítulo de una reputada monografía: Reinoso Suárez, Fernando. Development of the human diencephalon. In: Hassler, Rolf; Stephan, Hainz. Evolution of the Forebrain: Phylogenesis and ontogenesis of the forebrain. Stuttgart: Georg Thieme Verlag; 1966, p. 296-304.

54. Reinoso Suárez, Fernando. Correo electrónico al autor [Madrid], 2 de julio de 2011. Localizado en: Archivo del autor.

55. Diepen, R.; Engelhardt, F.; Smith-Agreda, V. Uber Ort und Art der Entstehung des Neurosecretes im Supraoptico-Hypophysaren System bei Hund und Katze. Verhandlungen der Anatomischen Gesellschaft (Anat. Anz. suppl.) 1954; 101: 276-286. 
-según afirmaba-, estaban «en íntima relación» con éste: los de Marburgo, Götingen, Hamburgo y, cómo no, el de el de Bargmann en Kiel ${ }^{56}$, muy especializado en el eje hipotálamo-hipofisario.

Por aquellos años, Spatz advirtió a Smith de que el laboratorio contaría en breve con material cerebral muy valioso de varias órdenes de mamíferos (lemúridos e insectívoros). Heinz Stephan, el responsable de la anatomía comparada de Giessen, había hecho un viaje al Congo y se había hecho con algunos ejemplares difíciles de conseguir. Animado por la noticia, el español se propuso completar sus investigaciones abordando un tema pendiente: la relación topográfica entre adeno y neurohipófisis desde el punto de vista filogenético ${ }^{57}$. Para ello solicitó ayuda económica a la Fundación Juan March, que había empezado el año antes a ofrecer becas.

A pesar de estas premisas comparativas, la embriología seguiría siendo para el anatomista un poderoso recurso en el que apoyar los nuevos datos. Tanta importancia daba a este aspecto que, antes de partir, se cercioró de que estuviera presente durante su estancia Werner Kahle, el neuroembriólogo del instituto, que se encontraba en EEUU en una estancia de investigación ${ }^{58}$.

Con estos objetivos, la March le concedió una beca ${ }^{59}$ con la que partió para la Alemania occidental. Su estancia, que se alargó desde el 15 de julio al 15 de octubre de 1957, resultó muy productiva y aportó interesantes observaciones a la evolución ontofilogénica del eje hipofisario. Describió cómo, a lo largo de la escala filogenética, pasaría progresivamente de ventro-caudal a ventro-oral - que es la posición en el hombre-, y demostró que la estructura evoluciona de la misma manera a lo largo del desarrollo embrionario.

En la misma línea, comenzó una investigación sobre son los llamados «vasos especiales»(Spezialgefässe), unas estructuras vasculares descritas en este sistema por la escuela alemana y que se planteaban como regula-

56. Smith Agreda, Víctor. Solicitud de Beca. Presupuesto económico aproximado, 1957. Localizado en: AFJM. Ayudas para investigaciones en el Extranjero, Grupo IV, 1957. Expediente de Víctor Smith Agreda.

57. Max Planck Institut für Hirnforschung [Probablemente de Spatz, aunque no aparece firma alguna]. Carta dirigida a Víctor Smith Agreda, Giessen, 19 de diciembre de 1956. Localizada en: AFJM. Extranjero, 1957. 526. Expediente del Becario Víctor Smith Agreda.

58. Max Planck Institut für Hirnforschung [Probablemente de Spatz, aunque no aparece firma alguna]. Carta dirigida a Víctor Smith Agreda, Giessen, 19 de diciembre de 1956. Localizada en: AFJM. Extranjero, 1957. 526. Expediente del Becario Víctor Smith Agreda.

59. Consejero secretario de la Fundación Juan March. Carta dirigida a Víctor Smith, Madrid, 6 de junio de 1957. AFJM. Extranjero, 1957. 526. Expediente de Víctor Smith Agreda. 
dores del eje. Durante su estancia en Giessen, pudo describir la evolución filogenética de dichos vasos e inició un estudio embriológico que posteriormente completó en España con el material que se iba acumulando en el departamento de Granada ${ }^{60}$.

A su vuelta, se materializó un viejo proyecto de Escolar: una sección de anatomía comparada. Esta rama de las ciencias morfológicas, tan olvidada por la ciencia española, tenía un inmenso interés para su anatomía. Por recomendación del propio Spatz, que envió una carta al catedrático español en el verano de $1955^{61}$, Escolar nombró jefe de la sección a Smith. Allí, prosiguió con la misma línea de investigación y estudió el sistema de comunicación de la hipófisis proximal en mamíferos inferiores y primates, eso sí, sin olvidar su correspondiente estudio embriológico.

\section{Conclusiones}

Las características sociopolíticas de la España de posguerra favorecieron la polarización de la anatomía en dos grandes escuelas que mantuvieron una estable hegemonía durante toda la Dictadura. El análisis de estos primeros años de Escolar y su equipo explica a la perfección su concepción del cuerpo humano y, en particular, las características de su embriología: descriptiva y fundamentadora de la anatomía macroscópica. Con los datos ofrecidos, se puede rastrear la base gegenbauriana de esta anatomía hasta la morfología dinámica practicada por Sánchez Guisande en Zaragoza antes de la guerra. La posterior estancia de Escolar en el laboratorio de Barcia le procuró no sólo el contacto con la anatomía brausiana, sino un teleogicismo particular que encajó muy bien en las bases ideológicas del Régimen. Este bagaje científico, unido a su militarización en el bando nacional durante la Guerra y a un catolicismo manifiesto, convirtieron a Escolar en el candidato ideal para ser apoyado por aquel primer CSIC de Albareda.

Así como los discípulos de Orts - lejos de la influencia del CSIC y con una financiación ajena al Estado- eligieron la Europa francófona y

60. Smith Agreda, Víctor. Memoria de la labor realizada por el Dr. D. Victor Smith Agreda durante el disfrute de una beca [...] en el Max Planck Institut de Giessen [...]. Localizada en: AFJM. Extranjero, 1957. 526. Expediente de Víctor Smith Agreda.

61. Spatz, Hugo. Carta a José Escolar. Giessen, 10 de agosto de 1955. Localizada en: AFJM. Extranjero, 1957. 526. Expediente de Víctor Smith Agreda. 
América como destino de sus estancias de investigación, los escolarianos, arrastrados por la política germanófila del Consejo, viajaron preferentemente a la RFA, donde estrecharon unos fuertes vínculos con el equipo de Hugo Spatz. Centraron su estudio en el cerebro y buscaron en la embriología y la filogénesis una explicación para la morfología del adulto. La embriología experimental, que estaba dotando de una gran reputación a la escuela rival, se mantuvo siempre alejada de sus intereses. La embriología se convirtió en uno más de los numerosos elementos divergentes de ambos grupos.

Los múltiples intereses adquiridos por Escolar en sus viajes (estereotaxia, morfología neuroendocrinológica, anatomía ontofilogenética...) se convirtieron progresivamente en verdaderas subespecialidades de las que se ocuparon sus discípulos. Esto explica por qué durante los años 50 sólo hemos mencionado a Reinoso y a Smith como cultivadores de la embriología y no han aparecido otros célebres miembros de la escuela como René Sarrat o Pedro Amat, muy especializados desde un primer momento. No obstante, la morfología de todos ellos se basa en el mismo núcleo escolariano, extremadamente holístico, por lo que todos mantuvieron siempre un gran interés por incorporar la embriología dentro de sus clases de anatomía.

A pesar de que tanto Reinoso como Smith terminaron abandonando la embriología, la esencia de la escuela permitió que durante la siguiente década los investigadores que se incorporaron al grupo - fundamentalmente José María Smith Agreda e Inés Fernández Ortega-, se especializaran en embriología y desarrollaran exitosas investigaciones en esta misma línea.

\section{Agradecimientos}

Este trabajo no hubiera sido posible sin el apoyo y orientación del Prof. Antonio Carreras Panchón, director de mi trabajo de investigación sobre la historia de la embriología española durante la II República y el Franquismo que presenté como tesis doctoral y de la que se extracta gran parte del presente artículo. 
